\title{
Fatores de Risco Associados a Doenças Cardiovasculares em Estudantes Universitários
}

\section{Risk Factors Associated with Cardiovascular Disease in College Students}

\author{
Raquel Oliveira Silva ${ }^{1}$ \\ Patrícia Vasconcelos Leitão Moreira² \\ Talita Maria Alves Lopes da Silva ${ }^{3}$ \\ Pamela Rodrigues Martins Lins ${ }^{4}$
}

\section{RESUMO}

Objetivo: Identificar os fatores de risco associados à doenças cardiovasculares em estudantes universitários. Metodologia: Trata-se de um estudo transversal realizado com 111 indivíduos com faixa etária entre 20 e 47 anos, numa residência universitária no Município de João Pessoa. Foram coletadas medidas antropométricas e realizada a aferição da pressão arterial. Além da estatística descritiva, foram aplicados os testes do Qui-quadrado de Pearson, para avaliar as associações entre as variáveis categóricas e o teste t de Student, para avaliar as diferenças entre as médias. Resultados: A frequência de hipertensão na amostra foi de $28,83 \%$. A circunferência do pescoço elevada foi positivamente associada ao sexo masculino $(p=0,025)$. Entre as medidas de adiposidade corporal, a obesidade geral, representada pelo índice de massa corporal $(p=0,042)$ e o percentual de gordura corporal $(p=0,021)$ demonstraram maior associação com a hipertensão. Na comparação entre as médias das variáveis antropométricas com a pressão arterial, foi observado que os indivíduos com a pressão arterial alterada também tinham o índice de massa corporal inadequado $(p=0,007)$, circunferência da cintura $(p=0,003)$, circunferência do pescoço $(p=0,014)$ e relação cintura/estatura $(p=0,012)$, sinalizando risco para doenças cardiovasculares. Conclusão: Dentre os fatores de risco associados à doenças cardiovasculares, foram observados alta frequência de hipertensão, excesso de peso, diâmetro do pescoço elevado no sexo masculino, enquanto, as medidas de adiposidade geral foram associadas ao aumento da pressão arterial.

\section{DESCRITORES}

Doenças Cardiovasculares. Hipertensão. Obesidade Abdominal. Antropometria.

\begin{abstract}
Objective: Identify risk factors associated with cardiovascular disease in college students. Methodology: This is a cross-sectional study conducted with 111 people aged between 20 and 47 years old in a university residence in the city of João Pessoa. Anthropometric measurements and blood pressure measurements were collected. In addition to descriptive statistics, Pearson's chi-square tests were applied to assess associations between categorical variables and Student's t test to assess differences between means. Results: The frequency of hypertension in the sample was $28.83 \%$. Elevated neck circumference was positively associated with males $(p=0.025)$. Among the measures of body adiposity, general obesity represented by the body mass index $(p=0,042)$ and the percentage of body fat $(p=0,021)$ demonstrated a greater association with hypertension. When comparing the means of anthropometric variables with blood pressure, it was observed that people with altered blood pressure also had an inadequate body mass index $(p=0,007)$, waist circumference $(p=0,003)$, neck circumference $(p=0,014)$ and waist/height ratio $(p=0,012)$, signaling risk for cardiovascular diseases. Conclusion: Among the risk factors associated with cardiovascular diseases, there was a high frequency of hypertension, overweightness, high neck diameter in males, while measures of general adiposity were associated with increased blood pressure.
\end{abstract}

\section{DESCRIPTORS}

Cardiovascular Diseases. Hypertension. Abdominal Obesity. Anthropometry.

${ }^{1}$ Nutricionista, Mestranda do Programa de Pós-Graduação em Ciências da Nutrição, Departamento de Nutrição da Universidade Federal da Paraíba, João Pessoa, Paraíba, Brasil.

${ }^{2}$ Pós-Doutora em Epidemiologia pela University of Liverpool, Inglaterra e docente do Curso de Nutrição, Departamento de Nutrição da Universidade Federal da Paraíba, João Pessoa, Paraíba, Brasil.

${ }^{3}$ Mestre em Ciências da Nutrição, docente do Curso de Nutrição, Departamento de Nutrição da Universidade Federal da Paraíba, João Pessoa, Paraíba, Brasil.

${ }^{4}$ Doutora em Ciências da Nutrição, docente do Curso de Nutrição, Departamento de Nutrição da Universidade Federal da Paraíba, João Pessoa, Paraíba, Brasil. 
egundo a Organização Pan-Americana da Saúde (OPAS), as Doenças cardiovasculares (DCVs) representam a principal causa de morte no mundo, sendo que três quartos desses casos acontecem em países de baixa e média renda, incluindo o Brasil e podem ser prevenidas com abordagem dos fatores comportamentais, que oferecem risco a população, como o tabagismo, as dietas inadequadas, o sedentarismo, a obesidade e o alcoolismo ${ }^{1}$. A partir deste cenário, a Organização Mundial da Saúde (OMS) coordena o Plano de ação global para a prevenção e o controle de doenças não transmissíveis 2013-2020 e planeja reduzir em $25 \%$ a carga de mortes por essa causa até $2025^{1}$.

A hipertensão e a obesidade são tidas como doenças multifatoriais predominantes em países de língua portuguesa, assim como tem sido demonstrado em outros países, sendo a hipertensão arterial sistêmica (HAS) uma patologia que representa um fator de risco para complicações que elevam as taxas de mortalidade, como o acidente vascular cerebral, o infarto agudo do miocárdio e a doença renal crônica².

Ao avaliar o perfil de risco para as doenças cardiovasculares entre universitários, Gonçalves et al. ${ }^{3}$ encontraram uma prevalência de $4,5 \%$ para a pressão sistólica e 4,4\% para a pressão diastólica, acima dos valores de normalidade em mulheres. Entre os homens a prevalência foi bem maior com $20 \%$ e 19,1\% para pressão sistólica e diastólica, respectivamente. Além disso, também observaram que $63,1 \%$ dos participantes tinham histórico familiar de HAS e $43,5 \%$ de hipercolesterolemia total, tidos como importantes fatores de risco modificáveis para DCVs.
Em pesquisas realizadas em todas as capitais brasileiras e o Distrito Federal, o Ministério da Saúde revelou que João Pessoa é a quarta capital da Região Nordeste que apresentou maior prevalência de obesidade entre indivíduos adultos ( $\geq 18$ anos de idade), com $20,4 \%$ da população obesa e $54,7 \%$ com excesso de peso ${ }^{4}$.

Tem-se utilizado indicadores antropométricos e circunferências corporais em estudos epidemiológicos para mensurar a adiposidade corporal e a distribuição da gordura corporal na associação com o risco cardiovascular. As mais utilizadas são o índice de massa corporal, a circunferência da cintura, do pescoço e a relação cintura-estatura ${ }^{5}$.

Existem evidências da elevação da prevalência de obesidade e hipertensão em indivíduos de todas as faixas etárias da população, a exemplo do Estudo de riscos cardiovasculares em adolescentes (ERICA) ${ }^{6}$. O presente estudo tem por objetivo identificar fatores de risco associados às doenças cardiovasculares em estudantes universitários.

\section{METODOLOGIA}

Trata-se de um estudo transversal realizado com indivíduos adultos de ambos os sexos, com faixa etária entre 20 e 47 anos, provenientes do interior do Estado da Paraíba e de outros estados, que vivem na condição de residentes na Universidade Federal da Paraíba (UFPB), Campus I, no Município de João Pessoa, contemplados com o auxílio residência universitária, da Pró-Reitoria de Assistência e Promoção ao Estudante (PRAPE).

A amostra foi composta por 111 voluntários dos 330 residentes. Participaram 
da pesquisa indivíduos de 9 dos 14 centros de ensino da universidade. Foram incluídos na amostra indivíduos adultos, de ambos os sexos, que moravam na residência universitária e estavam regularmente matriculados em cursos de graduação na modalidade presencial. Foram excluídos da amostra quatro participantes adolescentes, uma gestante e sete com dados incompletos no formulário aplicado. A amostragem se deu por conveniência, sendo elencados todos os participantes que atendessem os critérios de inclusão.

Os dados foram coletados por uma estudante do curso de graduação em nutrição da UFPB, no mês de maio de 2018. Para a coleta de dados foi utilizado um formulário para registro de dados sociodemográficos (idade, sexo e centro), pressão arterial e medidas antropométricas.

Para a obtenção do índice de massa corporal (IMC) foi coletada a estatura, com os participantes na posição ereta e na vertical, logo após a inspiração e com o auxílio de uma fita métrica inextensível fixada na parede sem rodapé. A coleta da variável peso foi realizada com os indivíduos utilizando roupas leves, sem objetos que pudessem interferir na medida e descalços em balança digital com escala de graduação para $100 \mathrm{~g}^{7}$.

A circunferência da cintura (CC) foi coletada com o participante ereto e abdômen relaxado, no ponto médio entre o último arco costal e a crista ilíaca, após o movimento expiratório, utilizando uma fita métrica inelástica ${ }^{8}$. Para o cálculo da relação cintura-estatura (RCE) foi utilizada a circunferência da cintura e a estatura, ambas em centímetros. A circunferência do pescoço (CP) foi coletada com uma fita inelástica com a cabeça do par- ticipante ereta, mediu-se horizontalmente logo acima da cartilagem cricotireóidea, abaixo da proeminência laríngea ${ }^{9}$.

Para a composição corporal, utilizou-se o método das 3 dobras cutâneas de Jackson e Pollock ${ }^{10}$ que sugere para os homens a dobra cutânea abdominal, mensurada na vertical, a dois centímetros da cicatriz do umbigo. A dobra da coxa foi obtida de forma paralela ao músculo reto femoral no ponto médio da coxa, entre o ponto de inserção do músculo e a parte superior da patela. A peitoral foi obtida no ponto médio da linha axilar ao mamilo, de forma oblíqua utilizando o adipômetro perpendicularmente ${ }^{11}$

Para as mulheres a dobra da coxa, a suprailíaca, foi obtida obliquamente, seguindo a linha axilar, $3 \mathrm{~cm}$ acima do ângulo superior do ílio (crista ilíaca) e a tricipital, coletada na vertical, no ponto médio entre o acrômio e o olecrano na face posterior do braço, sobre o músculo tríceps ${ }^{8}$. As medidas foram todas coletadas do lado direito dos voluntários, sendo repetidas três vezes, retirando-se as médias das mesmas, utilizando adipômetro científico Sanny ${ }^{\circledR}$, com faixa de medição de 0 a $65 \mathrm{~mm}$.

A pressão arterial sistólica (PAS) e pressão arterial diastólica (PAD) foram coletadas com um tensiômetro digital da marca Omron®, após repouso de 5 minutos, com o indivíduo sentado, sem ter fumado, consumido álcool ou bebida energética uma hora antes da aferição, além de não ter se exercitado nos 30 minutos prévios. A primeira aferição foi descartada, sendo utilizada a de maior valor após aferição nos dois membros ${ }^{2}$.

Para a obtenção da densidade corporal foi utilizado o somatório de 3 dobras pelo método proposto por Jackson e Pollock ${ }^{10} \mathrm{e}$ 
para determinação do \% percentual de gordura foi utilizada a fórmula de Siri ${ }^{11}$.

A determinação do estado nutricional foi realizada por meio do IMC (peso/altura²). Para a classificação do percentual de gordura corporal foi usada a referência de Lohman ${ }^{12} \mathrm{e}$ a classificação do estado nutricional ocorreu por meio da análise dos pontos de corte da $\mathrm{OMS}^{13}$. A CP foi considerada elevada para valores $\geq 39$ para homens e $\geq 35$ para mulheres ${ }^{14}$. Para a RCE foi considerado o ponto de corte $0,5^{15}$. A CC foi considerada normal quando inferior a $80 \mathrm{~cm}$ para mulheres e $94 \mathrm{~cm}$ para homens ${ }^{16}$.

Os pontos de corte utilizados para classificar a pressão arterial foram os do Guideline for theprevention, detection, evaluation, and management of high bloodpressure in adults ${ }^{17}$ que considera normais os valores de PAS e PAD $<120 \mathrm{mmHg}$ e $<80 \mathrm{mmHg}$, respectivamente e elevados PAS $>120 \mathrm{mmHg}$ e a PAD normal. Para hipertensão, $130-139 \mathrm{mmHg}$ para PAS ou 80-89 $\mathrm{mmHg}$ para PAD no estágio I, enquanto no estágio $\| \geq 140 \mathrm{mmHg}$ para a PAS ou $\geq 90 \mathrm{mmHg}$ para a $P A D^{17}$.

A partir dos pontos de corte de Lohman $^{12}$ a classificação do percentual de gordura corporal foi estratificada em três variáveis: reduzido, para aqueles que ficaram abaixo da média ou em risco de doenças e desordens associadas à desnutrição; adequado, para aqueles que ficaram na média e elevado para aqueles que ficaram acima da média ou em risco de doenças associadas à obesidade.

Para o IMC, os tipos de obesidade foram considerados como uma só variável, o mesmo ocorreu com a desnutrição. Na pres- são arterial, todos os valores encontrados acima do ponto de corte para normalidade (< $120 \mathrm{mmHg}$ PAS e < $80 \mathrm{mmHg}$ PAD) de acordo com a Guideline for theprevention, detection, evaluation, and management of high bloodpressure in adults ${ }^{17}$, foram incluídas em uma só variável, considerando como alterada.

O processamento dos dados se deu a partir do recurso Microsoft Office Excel 2013 e, em seguida, foi exportado para o programa estatístico STATA (Statistics/Data Analysis) versão 14.0 , para se proceder à análise estatística.

Para tanto, foi utilizada a estatística descritiva e inferencial. Na estatística descritiva, as variáveis quantitativas foram descritas em média e desvio-padrão, frequências absolutas e relativas. Após a execução do teste de normalidade dos dados (Teste de Komolgorov-Smirnov) foram realizados testes de correlação paramétricos. As associações entre as variáveis categóricas foram avaliadas pelo teste Qui quadrado de Pearson. As comparações entre as médias foram realizadas pelo teste t de Student. Para todas as análises adotou-se a significância de 0,05.

O estudo foi aprovado pelo Comitê de Ética do Centro de Ciências da Saúde da Universidade Federal da Paraíba, Protocolo $n^{\circ}$. 88158218.8.0000.5188 e foram seguidas as orientações da Resolução n ${ }^{\circ}$ 466/2012, que regulamenta as pesquisas com seres humanos. Os indivíduos com diagnóstico nutricional desfavorável e/ou PA alterada foram aconseIhados a procurar os serviços do Centro de Referência em Atenção à Saúde (CRAS) da Universidade Federal da Paraíba ou a Unidade de Saúde da Família mais próxima. 


\section{RESULTADOS}

Dos 14 centros acadêmicos que compõem o Campus I, foram avaliados indivíduos de 9 centros, sendo a maior participação do Centro de Ciências Humanas Letras e Artes, com 24 voluntários.

Dos 111 indivíduos avaliados, 63,96 $\%$ eram do sexo masculino, com faixa etária média de 24,22 $\pm 0,37$ anos. O IMC médio da amostra foi de $23,53 \pm 0,40 \mathrm{~kg} / \mathrm{m}^{2}$, enquanto o percentual de gordura corporal médio totalizou 16,90 \pm 0,69 \%. Quando se observou a distribuição por frequência, notou-se que aproximadamente $30 \%$ da amostra encontrava-se com excesso de peso (sobrepeso $25,23 \%$ e obesidade $5,41 \%$ ) e cerca de $37 \%$ com percentual de gordura corporal elevado, sendo acompanhado pela CP $(41,44 \%)$, RCE $(30,63 \%)$ e a PA $(28,83 \%)$ elevados. Não foi encontrada relação estatisticamente significativa entre as variáveis sexo e a pressão arterial, porém, foi registrada maior prevalência de PA alterada entre os homens, conforme a Figura 1.

A Tabela 1 expõe a associação entre as variáveis antropométricas e o sexo. Pode-se observar que os homens apresentaram maiores percentuais de excesso de peso e RCE mais desfavorável que as mulheres, ainda que estas relações não tenham sido significativas estatisticamente, mas representam fatores associados às doenças cardiovasculares. Entretanto, esta relação foi estatisticamente significativa quando se associou a CP com o sexo $(p=0,025)$.

$\mathrm{Na}$ associação entre as variáveis relacionadas à mensuração da gordura geral (IMC, percentual de gordura corporal) e gordura central (RCE, CP e CC) com a PA (Tabela 2), foi constatada associação estatisticamente significativa entre o IMC $(p=0,042)$, o percentual de gordura corporal $(p=0,021)$ e a PA alterada.

Ao comparar as médias entre a PA e as variáveis antropométricas, foi observado

Figura 1. Frequência da pressão arterial por sexo entre estudantes de uma residência universitária no Município de João Pessoa-PB, 2018

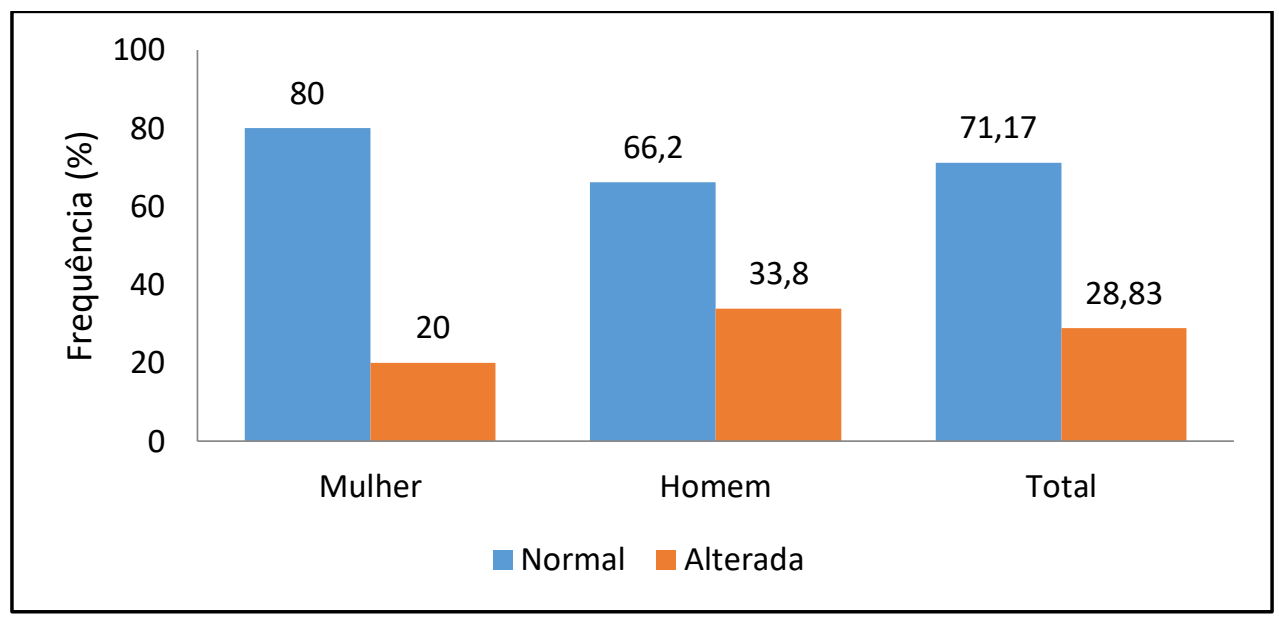

Fonte: pesquisa. Valor de $p=0,123$ 
Tabela 1. Distribuição por sexo das variáveis antropométricas e de outros indicadores de estudantes de uma residência universitária no Município de João Pessoa-PB, 2018

\begin{tabular}{|c|c|c|c|c|c|c|c|}
\hline & \multicolumn{2}{|c|}{ Feminino } & \multicolumn{2}{|c|}{ Masculino } & \multicolumn{2}{|c|}{ Total } & \multirow[b]{2}{*}{$p^{*}$} \\
\hline & $\mathrm{n}$ & $\%$ & $\mathrm{n}$ & $\%$ & $\mathrm{n}$ & $\%$ & \\
\hline \multicolumn{8}{|l|}{ IMC } \\
\hline Baixo peso & 05 & 12,50 & 05 & 7,04 & 10 & 9,01 & \multirow[t]{4}{*}{0,120} \\
\hline Adequado & 28 & 70,00 & 39 & 54,93 & 67 & 60,36 & \\
\hline Sobrepeso & 05 & 12,50 & 23 & 32,39 & 28 & 25,23 & \\
\hline Obesidade & 02 & 5,00 & 04 & 5,63 & 06 & 5,41 & \\
\hline \multicolumn{8}{|l|}{ CC } \\
\hline Adequada & 29 & 72,50 & 61 & 85,92 & 90 & 81,08 & \multirow[t]{2}{*}{0,083} \\
\hline Risco & 11 & 27,50 & 10 & 14,08 & 21 & 18,92 & \\
\hline \multicolumn{8}{|l|}{ CP } \\
\hline Adequada & 29 & 72,50 & 36 & 50,70 & 65 & 58,56 & \multirow[t]{2}{*}{$0,025^{\star *}$} \\
\hline Risco & 11 & 27,50 & 35 & 49,30 & 46 & 41,44 & \\
\hline \multicolumn{8}{|l|}{ RCE } \\
\hline Adequada & 30 & 75,00 & 47 & 66,20 & 77 & 69,37 & \multirow[t]{2}{*}{0,334} \\
\hline Risco & 10 & 25,00 & 24 & 33,80 & 34 & 30,63 & \\
\hline \multicolumn{8}{|c|}{ Gordura Corporal (\%) } \\
\hline Reduzido & 19 & 47,50 & 44 & 61,97 & 63 & 56,76 & \multirow[t]{3}{*}{0,335} \\
\hline Adequado & 03 & 7,50 & 04 & 5,63 & 07 & 6,31 & \\
\hline Elevado & 18 & 45,00 & 23 & 32,39 & 41 & 36,94 & \\
\hline
\end{tabular}

Fonte: pesquisa. IMC (Índice de Massa Corporal); CC (Circunferência de cintura); CP (Circunferência de pescoço); RCE (Relação cintura/estatura). * Qui quadrado de Pearson. ${ }^{* *}$ estatisticamente significativo, $p<0,05$

Tabela 2. Associação entre os parâmetros de adiposidade geral e central com a pressão arterial entre estudantes de uma residência universitária no Município de João Pessoa-PB, 2018

\begin{tabular}{|c|c|c|c|c|c|}
\hline & & lormal & $\mathrm{PA}$ & erada & \\
\hline & $\mathrm{n}$ & $\%$ & $\mathrm{n}$ & $\%$ & $\mathrm{p}^{*}$ \\
\hline IMC & & & & & \\
\hline Baixo peso & 09 & 90,00 & 01 & 10,0 & $0,042^{* *}$ \\
\hline Adequado & 51 & 76,12 & 16 & 23,88 & \\
\hline Sobrepeso & 17 & 60,71 & 11 & 39,29 & \\
\hline Obesidade & 02 & 33,33 & 04 & 66,67 & \\
\hline CC & & & & & \\
\hline Adequada & 65 & 72,22 & 25 & 27,78 & 0,613 \\
\hline Risco & 25 & 27,78 & 07 & 33,33 & \\
\hline $\mathrm{CP}$ & & & & & \\
\hline Adequada & 47 & 72,31 & 18 & 27,69 & 0,753 \\
\hline Risco & 32 & 69,57 & 14 & 30,43 & \\
\hline RCE & & & & & \\
\hline Adequada & 57 & 74,03 & 20 & 25,97 & 0,318 \\
\hline Risco & 22 & 64,71 & 12 & 35,29 & \\
\hline Gordura Co & & & & & \\
\hline Reduzido & 51 & 64,56 & 12 & 37,50 & $0,021^{\star *}$ \\
\hline Adequado & 03 & 3,80 & 04 & 12,50 & \\
\hline Elevado & 25 & 31,65 & 16 & 50,00 & \\
\hline
\end{tabular}


que os indivíduos com a PA alterada também apresentaram IMC $(p=0,007), C C(p=0,003)$, CP $(p=0,014)$ e RCE $(p=0,012)$ inadequados, indicando risco para DCV. Não existiu associação estatisticamente significativa entre as médias da PA e do percentual de gordura corporal.

\section{DISCUSSÃO}

O presente estudo buscou identificar os fatores associados as DCVs em estudantes universitários que residem em uma residência universitária e são assistidos pela assistência estudantil. Foi constatado que aproximadamente um terço da amostra se encontrava com excesso de peso $(25,23 \%$ com sobrepeso e $5,41 \%$ com obesidade) e $36,93 \%$ com percentual de gordura corporal elevado.

El Zein et al. ${ }^{18}$ compararam medidas antropométricas de universitários e identificaram que os alunos com segurança alimentar marginal (incerteza e ansiedade relacionadas ao suprimento de alimentos) tinham 3 vezes mais chances de serem obesos, enquanto os alunos com insegurança alimentar, as chances eram 5 vezes maiores, destacando que em face às despesas, os estudantes priorizam alimentos mais baratos, porém, mais densos em energia.

Ao avaliar o consumo alimentar de universitários que moram ou não com os pais, Barbara e Ferreira-Pêgo ${ }^{19}$ identificaram diferenças entre os grupos sobre o consumo de peixes $(p<0,001)$, legumes $(p<0,002)$ e fastfoods ( $p<0,027)$. Os alunos que vivem fora do núcleo familiar consumiam peixes com menor frequência e os fastfoods e os legumes com maior frequência, enquanto, os alunos que vivem com os pais, mantiveram a frequência de consumo destes alimentos ${ }^{19}$. Em outro es- tudo com universitários, observou-se que um quarto dos participantes não tomava o café da manhã e menos de $10 \%$ não almoçavam ${ }^{20}$.

Cabe ressaltar que a presente amostra é composta exclusivamente por universitários, provenientes de cidades que não incluem o Município de João Pessoa (localização do Campus) e a região metropolitana. Portanto, são estudantes que vivem fora do convívio familiar e, assim, os seus hábitos alimentares podem sofrer alterações.

A frequência de hipertensão na amostra alcançou $28,83 \%$, semelhante à verificada por Ware et al. ${ }^{21}$, em indivíduos com idade entre 18 e 49 anos, 28\% foram classificados como hipertensos. A associação entre a $\mathrm{CP}$ e o sexo evidenciou, apenas nos homens, significância estatística, ressaltando que este público possui maior deposição de gordura nesta região. Pereira et al. ${ }^{14}$ ao analisarem a relação entre a $\mathrm{CP}$ e o sexo em universitários, obtiveram resultado semelhante, a quantidade de indivíduos que apresentaram medidas da CP acima dos valores de normalidade foi maior no sexo masculino, sendo ainda associadas a componentes da síndrome metabólica.

Téllez et al. ${ }^{22}$ avaliaram a associação entre a $\mathrm{CP}$, outros indicadores antropométricos, como o IMC, a CC e os indicadores de composição corporal por Absorciometria de raio-X Dupla (DXA), em jovens adultos espanhóis. Foi observada uma associação entre a $\mathrm{CP}$ e todos os indicadores, porém, o IMC e a CC demonstraram ser melhores preditores de gordura central e total.

Embora sem significância estatística neste estudo, em outros estudos com universitários, foi demonstrado que o número de homens com sobrepeso, obesidade e PA elevada foi maior em relação às mulheres. A exemplo de Zhang et al. ${ }^{23}$ que observaram níveis 
maiores de IMC, CC e PA entre os homens, além da associação entre o IMC e o aumento da PA. Também foram encontrados valores superiores nas CC, circunferência do quadril, RCE, IMC, PAS e prevalência de hipertensão entre os homens. As mulheres apresentaram valores superiores referentes à quantidade de gordura corporal. Em ambos os grupos foi observado que os participantes hipertensos possuíam IMC mais elevado ${ }^{24}$. Esse fato traz preocupação porque em ambos os sexos, o excesso de peso se relacionou a hipertensão. Sendo estes jovens universitários geralmente usuários do restaurante universitário, ressalta-se a necessidade de um acompanhamento nutricional pelo CRAS, para que seja realizada uma educação nutricional com modificação de hábitos alimentares.

$\mathrm{Na}$ avaliação das variáveis de adiposidade na predição da hipertensão, os indicadores de obesidade geral demonstraram maior associação no aumento da prevalência de hipertensão. Em uma recente revisão com meta-análise foi encontrado uma relação dose-resposta entre diferentes medidas de adiposidade e o risco de hipertensão. Foi estabelecida uma associação entre o aumento de $5 \mathrm{~kg} / \mathrm{m}^{2}$ no IMC com aumento de $50 \%$ no risco de hipertensão, um incremento de $10 \mathrm{~cm}$ na CC com aumento de $25 \%$ e aumento de 0,1 na RCE, representando $27 \%$ no risco de incidência de hipertensão ${ }^{25}$, sinalizando que o IMC estabelece uma relação mais forte.

Em um estudo de séries temporais com universitários foi estabelecido à seguinte proporção entre as variáveis de adiposidade geral e central com a hipertensão, que o aumento de $1 \mathrm{~kg} / \mathrm{m}^{2}$ no IMC foi relacionado a uma elevação de $11 \%$ na prevalência de hipertensão. O aumento de $1 \mathrm{~cm}$ na $\mathrm{CC}$ demonstrou associação com um aumento de $9 \%$ na prevalência de hipertensão ${ }^{26}$.

Sabe-se que quando em excesso, a gordura visceral é produtora de citocinas responsáveis por cascatas de reações infamatórias e estresse oxidativo, por vezes, relacionados à resistência à insulina, disfunção endotelial e rigidez vascular. Com isso, a expressão do fator de necrose tumoral-alfa (TNF- $\alpha$ ) estimula a secreção de leptina, a qual possui papel na hiperatividade simpática associada a hipertensão ${ }^{27}$.

Além disso, a hiperatividade do sistema renina-angiotensina-aldosterona tem sido observada como resultante do tecido adiposo disfuncional característico na obesidade. Estas alterações refletem no aumento da reabsorção renal de sódio e pressão natriurese prejudicada em conjunto com os efeitos simpatoexcitatórios da angiotensina II, como alterações microvasculares (responsável pela resistência vascular periférica), em face da síntese reduzida de óxido nítrico derivado do endotélio, gerando vasoconstrição e aumento da pressão arteriall $^{28}$.

Os indivíduos que apresentaram valores inadequados de IMC, CC, CP e RCE também apresentaram níveis pressóricos alterados, corroborando com o estudo de Famodu et al. ${ }^{29} \mathrm{em}$ que a CP apresentou correlação positiva com IMC, CC, RCE e PAS ( $p<$ 0,0001 ) em ambos os sexos e ainda no caso das mulheres com a PAD ( $p<0,0001)$. Fantin et al. ${ }^{30}$ verificaram em sua amostra composta por indivíduos com sobrepeso e obesidade, que aqueles com a CP elevada também apresentavam maiores níveis de PAS, PAD e PAM, além de resistência à insulina e menores taxas de colesterol HDL-c. A CP elevada também foi associada a piores valores de rigidez arterial em relação aos indivíduos que tinham as $\mathrm{CP}$ normais. 
Apesar da desproporcionalidade entre o número de participantes por sexo, onde os indivíduos do sexo masculino se encontraram em maior número em relação ao sexo feminino na amostra, o que poderia ser visto como uma limitação. Por outro lado, como potencialidade, destaca-se o fato da pesquisa ser realizada no fim do período letivo, onde os estudantes encontravam-se adaptados a sua rotina e expostos as características que compõem o perfil da amostra.

Outros estudos podem ainda ser realizados utilizando protocolos que possam definir outros fatores de risco, a exemplo de estudos longitudinais que incluam análise do consumo alimentar, a prática de atividade física, a qualidade do sono e os parâmetros bioquímicos.

\section{REFERÊNCIAS}

1. Organização Pan Americana de Saúde/Organização Mundial da Saúde/Brasil. Doenças Cardiovasculares. 2017 [cited 04 Mar 2018].

2. Oliveira GMM, Mendes M, Malachias MVB, Morais J, Moreira FO, Coelho AS et al. Diretrizes de 2017 para manejo da hipertensão arterial em cuidados primários nos países de língua portuguesa. Rev Portuguesa de Cardiologia. 2017; 36(11):789-798.

3. Gonçalves JS, Silva LL, Ninahuaman MFML, Ab Dala GA, Lima MOP. Perfil de Risco Cardiovascular em Estudantes Universitários. Life Style. 2018, 5(2): 91-108.

4. Brasil. Ministério da Saúde. Vigitel Brasil 2019: vigilância de fatores de risco e proteção para doenças crônicas por inquérito telefônico: estimativas sobre frequência e distribuição sociodemográfica de fatores de risco e proteção para doenças crônicas nas capitais dos 26 estados brasileiros e no Distrito Federal em 2019 /Ministério da Saúde, Secretaria de Vigilância em Saúde. Departamento de Vigilância de Doenças e Agravos não Transmissíveis e Promoção da Saúde Brasília, DF: [s.n.], 2020.

5. Santos EGR, Pereira PY, Sekiya DRU,Goulart RMM. Prevalência de risco cardiovascular a partir de parâmetros antropométricos em crianças e adolescentes. Rev de Atenção à Saúde. 2019; 17(60):54-62.

\section{CONCLUSÃO}

A partir dos achados do presente estudo pode-se concluir que um perfil nutricional desfavorável refletiu na frequência de hipertensão entre jovens adultos universitários, moradores em uma residência universitária. $A$ adiposidade corporal tem um papel fundamental no aumento da PA. Foram encontradas medidas de CC, CP, RCE e IMC adequadas com os níveis pressóricos normais. Além disso, as medidas de adiposidade geral elevadas foram associadas à hipertensão, enquanto, a CC elevada foi mais observada no sexo masculino. Mais estudos são recomendados com este público universitário.

6. Bloch KV, Klein CH, Szklo M, KuschnirMCC, Abreu GDA Barufaldi LA, et al. ERICA: prevalências de hipertensão arterial e obesidade em adolescentes brasileiros. Rev. Saúde Pública. 2016: 50 Suppl 1.

7. Pazin DC, Rosaneli CF, Olandoski M, Oliveira ERN, Baena CP, Figueredo AS et al . Circunferência da Cintura está Associada à Pressão Arterial em Crianças com Índice de Massa Corpórea Normal: Avaliação Transversal de 3417 Crianças Escolares. Arq. Bras. Cardiol. 2017; 109(6):509515.

8. Silveira EA, Pagotto V, Barbosa LS, Oliveira C, Pena GG, Velasquez-Melendez G. Acurácia de pontos de corte de IMC e circunferência da cintura para a predição de obesidade em idosos. Ciência \& Saúde Coletiva. 2020; 25(3):1073-1082.

9. Küçük U, Küçük HO, Cüce $F$, Balta $S$. Relationship between neck circumference and epicardial fat thickness in a healthy male population. Arq Brasileiros de Cardiologia. 2016; 107(3): 266-270.

10. Jackson AS, Pollock ML. Generalized equations for predicting body density of men. British J Nutrition. 1978 40(3):497-504 
11. Freitas, MAFI, Santos ATC, Lucena JS, Takenami IO, Rezende MOC. Percentual de gordura corporal de jogadores de futebol. RBNE- Rev Bras Nutrição Esportiva. 2017; 11(65):603-609.

12. Lohman T. Advances in Body Composition Assessment: Current Issues in Exercise Science series. Monograpf $\mathbf{n}^{\circ}$ 3. Champaign, Illinois: HumanKinetics, 1992.

13. World Health Organization. Obesity. Preventing and Managing the Global Epidemic. Report of a WHO Consultation on Obesity. World Health Organization, Geneva. 1997: 107-158.

14. Pereira DCR, Araújo MFM, Freitas RWJF, Souza TCR, Zanetti ML, Damasceno MMC. Circunferência do pescoço como possível marcador para síndrome metabólica em universitários. Rev Latino-Americana de Enfermagem. 2014; 22(6):973-979.

15. ABESO - Diretrizes brasileiras de obesidade 2016 - Associação Brasileira para o Estudo da Obesidade e da Síndrome Metabólica. 2016; 4: 07-188.

16. Malachias MVB, Souza WKSB, Plavnik FL, Rodrigues CIS, Brandão AA, Neves MFT, et. al. VII Diretriz Brasileira de Hipertensão Arterial. Arq Bras Cardiol. 2016; 107(3):1-83.

17. Whelton PK, Carey RM, Aronow WS, Casey DE Jr, Collins KJ, Dennison HC, et al. ACC/AHA/AAPA/ABC/ACPM/ AGS/APhA/ASH/ASPC/NMA/PCNA guideline for the prevention, detection, evaluation, and management of high blood pressure in adultsg: a report of the American College of Cardiology/American Heart Association Task Force on Clinical Practice Guidelines. J American College of Cardiology. 2017:481.

18. El Zein A, Colby SE, Zhou W, Shelnutt KP, Greene GW, Horacek TM, Olfert MD, Mathews AE. A insegurança alimentar está associada ao aumento do risco de obesidade em estudantes universitários dos EUA. Curr Dev Nutr. 2020, 4(8).

19. Bárbara R, Ferreira-Pêgo C. Mudanças nos hábitos alimentares de universitários deslocados e não deslocados. Int J Environ Res Saúde Pública. 2020; 17(15): 5369.

20. Porto-Arias JJ, Lorenzo T, Lamas A, Regal P, Cardelle-Cobas A, Cepela A. Padrões alimentares e avaliação nutricional em universitários galegos. J Physio IBiochem. 2018; 74 (1):119-126.

21. Ware LJ, Charlton K, Schutte AE, Cockeran M, Naidoo N, Kowal P. Associações entre sal dietético, potássio e pressão arterial em adultos sul-africanos: WHO SAGE Wave 2 Salt \&Tobacco. Nutrição, Metabolismo e Doenças Cardiovasculares. 2017; 27(9):784 -791.
22. Arias Téllez MJ, Acosta FM, Sanchez-Delgado G, Martinez-Tellez B, Muñoz-Hernández V, Martinez-Avila WD, Henriksson P, Ruiz JR. Association of Neck Circumference with Anthropometric Indicators and Body Composition Measured by DXA in Young Spanish Adults. Nutrients. 2020,12(2):514.

23. Zhang Y, Wang S, Zhao J, Chu Z. Prevalência de sobrepeso e obesidade central e sua relação com a pressão arterial em estudantes universitários de Shandong, China. Blood Pressure Monitoring. 2016; 21(4): 251-154.

24. Ilow R, Różańska D, Regulska-llowB. Prevalence of cardiovascular disease risk factors among pharmacy students from Wroclaw Medical University (Poland). AdvClinExp Med. 2017; 5(26): 843-850.

25. Zhou W,Shi1 Y, Li Y, Ping Z,Wang C, Liu X, et al. Índice de massa corporal, gordura abdominal e incidência de hipertensão: meta-análise dose-resposta de estudos prospectivos. J Human Hypertension. 2018; 32(5):321 333.

26. Choukem SP, Kengne AP, Nguefack ML, Mboue-Djieka Y, Nebongo D, GuimezapJ,et al. Four-year trends in adiposity and its association with hypertension in serial groups of young adult university students in urban Cameroon: a time-series study, BMC Public Health. 2017; 499 (17):1-6.

27. Faria AP, Ritter AMV, Gasparetti CS, Corrêa NB, Brunell V, Almeida A et al . Proposta de um Escore Inflamatório de Citocinas e Adipocinas Plasmáticas Associado à Hipertensão Resistente, mas Dependente dos Parâmetros de Obesidade. Arq. Bras. Cardiol. 2019; 112 (4):383-389.

28. Schutten MT, Houben AHM, Leeuw PW, Stehouwer CDA A ligação entre a sinalização do Sistema Renia-Angiotensina-Aldosterona do tecido adiposo e a hipertensão associada à obesidade. Physiology. 2017; 32(3):197-209.

29. Famodu OA, Barr ML, Colby SE, Zhou W, Holásková I, Leary MP, Byrd-Bredbenner C, Mathews AE, Olfert MD. A circunferência do pescoço se relaciona positivamente com fatores de risco cardiovascular em estudantes universitários. Int J Environ Res Saúde Pública. 2018; 15(7):1480

30. Fantin F, Comellato G, Rossi AP, Grison E, Zoico E, Mazzali $G$, et al. Relação entre circunferência do pescoço, resistência à insulina e rigidez arterial em indivíduos com sobrepeso e obesidade. European Association Preventive Cardiology. 2017; 24(14):1532-1540.

\section{CORRESPONDÊNCIA}

Raquel Oliveira Silva

Rua Diogo Velho, 231, Apto 105, Centro,

João Pessoa, Paraíba, Brasil, CEP: 58013-110.

Email: raquelipec@hotmail.com 